\title{
A framework for maintenance 4.0
}

\author{
Erkki Jantunen*, Giovanni Di Orio**, Felix Larrinaga***, Martin Becker****, Michele Albano*****, and \\ Pedro Maló** \\ *VTT Technical Research Centre of Finland Ltd, P.O. Box 1000, 02044 VTT, Finland, email: erkki.jantunen@vtt.fi \\ **Dep De Eng. Eletrotécnica, FCT-UNL, UNINOVA-CTS, Lisboa, Portugal \\ ***Mondragon University, Loramendi 4, 20500 Mondragon, Spain \\ ****Fraunhofer IESE, Fraunhofer Platz 1, 67663 Kaiserslautern, Germany \\ *****CISTER, ISEP/INESC-TEC, Polytechnic Institute of Porto, 4249-015 Porto, Portugal
}

\begin{abstract}
The rapid development of new low-cost sensors of reasonable processing power have led to the introduction of Cyber-Physical Systems (CPS) to support maintenance, which in turn together with a scalable, two level data processing architecture has taken maintenance as such to a new level i.e. Maintenance 4.0. The potential of CPS to support maintenance is explained, and it is related to the requirements set upon the sensors, such as robustness, communication capabilities, intelligence, small size, etc. A new framework and architecture with support for Health management, Prognostics and Collaborative decision-making functionalities that takes the full advantage of the above introduced new technologies is described both theoretically and in the light of some industrial use case examples. A comparative between the approaches implemented in the use cases brings light into the maintenance possibilities to address in such a framework.
\end{abstract}

\section{Introduction}

The combination of new emerging technologies and paradigms such as IoT, Machine-to-Machine (M2M), Cloud/Edge computing, service-oriented architecture (SOA), service-oriented computing (SOC) together with the wider dissemination of information technology (IT) and embedded technologies are driving powerful and, above all, new business opportunities for enterprises. As explained by Di Orio et al. (2015) current technological trends are radically changing the way systems in different domains like manufacturing, healthcare, transportation, consumer electronics, etc. are designed and deployed, as well as, monitored and controlled. In particular, to capitalize on the key markets opportunities and winning the competition for markets share, manufacturing companies are caught between the growing needs for: a) implementing more and more exclusive, efficient and sustainable production systems to assure a more efficient and effective management of the resources and to produce innovative and appellative customized products as quickly as possible with reduced costs while preserving product quality; and $b$ ) creating new sources of value by providing new integrated product-service solutions to the customer (Cavalieri et. al. 2012). Future internet technologies such as Internet of Things (IoT) - supported by the wider dissemination of Cyber-Physical Systems (CPS) facilitate the deployment of advanced solutions in plant floor, as well as, applications and services while promoting the meshing of virtual and physical devices and the interconnection of products, processes, infrastructures and more closely connected customers, suppliers and manufacturers within the manufacturing value chain. As stated in Acatech (2011), three main converging trends are pushing manufacturing enterprise into the world of "smartness \& connectivity", namely: 1) Smart embedded systems, mobile services, and pervasive computing (IoT paradigm); 2) Internet based business processes; and 3) social network and communities. In particular, smart embedded systems, mobile services and pervasive computing are contributing to the establishment of a cyber space where data coming from asset and resources can be potentially used to provide some added value. As a parallel to the emerging "Industry 4.0", which is the term coined for this industrial (r)evolution, a number of subfield have been created, between them "Maintenance 4.0", which comprises all topics related to the application of Industry 4.0's techniques and technologies to maintenance. This paper is grounded upon one of these European research initiatives the ECSEL-MANTIS project and describes how it aims at revolutionising maintenance by means of CPS, to attain Maintenance 4.0.

\section{Components of Cyber-Physical Systems}

Nowadays, the research stream on CPS is extremely active and vibrant as also confirmed by the number of research activities on the topic. As a matter of fact, there is extensive literature dealing with the materialization of the CPS vision and the related challenges technical, social and educational as confirmed in Bytschkow et 
al. (2014). As stated in Leitão et al. (2016), the term "Cyber-Physical Systems" was coined in 2006, but several definitions of the term can be found in the literature. According to Baheti et al. (2011) CPS can be defined as transformative technologies that allow to manage interconnected systems between physical assets and computational capabilities. The definitions in Steering Committee (2013) highlight the concept of collaboration and service provisioning. A working definition for CPS has been offered By Cengarle et al. (2013), where a CPS is defined as a system consisting of computational, communication and control components combined with physical processes. Regardless to the specific definition of CPS it is possible to identify their core elements and/or characteristics, extended from Sanislav et al. (2012): 1) Enhancement of physical entities with Cyber capabilities; 2) Networked at multiple and extreme scale; 3) Dynamic behaviour (plug and unplug during operation); 4) High degrees of automation, the control loops are typically closed; 5) High degree of autonomy and collaboration to achieve a higher goal; and 6) tight integration between devices, processes, machines, humans and other software applications. Connected to the term CPS several other terms have been created for better express and defining groups and networks of connected CPSs.

\section{Service Oriented Architecture for Cyber Physical Systems}

The Device Profile for Web Services (DPWS) has been designed and developed to introduce web services protocols for device networking (Cândido, 2013). It is a standard supported by the OASIS Web Services Discovery and Web Services Devices Profile Technical Committee, since June 2009. DPWS is a stack of webbased protocols and profile for devices, which defines two fundamental elements: the device and its hosted services. Devices perform the discovery and metadata exchange protocols, and hosted services (developed by end-users) provide the functional behaviour of the device DPWS specifies also a set of infrastructure services: a) Discovery services (WS-Discovery): used by a device connected to a network to advertise itself and to discover other devices (Oasis, 2009). WS-Discovery uses User Datagram Protocol (UDP) and a multicast address to broadcast and listen to the discovery messages. b) Metadata exchange services (WS-Metadata Exchange): provide dynamic access to de- vices hosted services and to their metadata, such as WSDL or XML Schema definitions. c) Event publish/subscribe services (WS-Eventing): allow other devices to subscribe to asynchronous messages (events) produced by a given vendor-defined service. DPWS is built on top of the SOAP 1.2 standard, and relies on additional WS specifications, such as WS-Addressing and WS-Policy, to further constrain the SOAP messaging model. At the highest level, the messages correspond to vendor-specific actions and events. Messages are delivered using HTTP, Transmission Control Protocol (TCP) and UDP transport protocols. More details about DPWS can be found in Rajaraajeswari et al. (2016). Due to its popularity, considerable open and not open implementations of DPWS can be found, the most relevant are: WS4D Java Multi Edition DPWS Stack (JMEDS) or the Microsoft Web Service on Device API (WSDAPI). The OPC UA (Unified Architecture) is the new version of the vastly deployed OPC architecture originally designed by the OPC Foundation to connect industrial devices to control and supervision applications as explained by Hadlich (2006). The focus of OPC is on getting access to large amounts of real-time data while ensuring performance constraints without disrupting the normal operation of the devices. The original OPC specifications were based on Microsoft COM/DCOM. The main visible transformation when comparing OPC with and its newer architecture OPC-UA is the shift to cross-platform capable web services (Candido et al. 2010). Moreover, OPC-UA includes support for secure communication, support for the publish-subscribe communication paradigm, unification of several OPC data models as a single set of services and the extension to other domains such as manufacturing (shop-floor), production, maintenance, and business application to implement the total integrated manufacturing. OPC-UA can be mapped using widespread Web standards, including XML, WSDL, SOAP and other WS-* specifications, along with other OPC UA defined specifications. To facilitate and spread as much as possible the usage of OPC-UA technology and strategy/approach the OPC-UA community is collaborating with major industry standards organizations as well as to the possibility to move the information models provided by these organizations to the end user community. Finally, OPC UA provides a homogeneous and generic meta-model and defines a set of web services interfaces to represent and access both structure information and state information in a wide range of devices. The Arrowhead Framework (Delsing et al. 2017) is the result of a large European effort that aimed at normalizing the interaction between industrial IoT applications by means of Service Oriented Architectures. 
The approach targeted a number of application domains comprising industrial production, smart buildings, electro mobility, and energy production. The services are exposed and consumed by (software) systems, which are executed on devices. The devices are grouped into local automation clouds, which are self-contained, geographically co-located, independent from each other, and protected from external access through security measures. Services are considered either application services when implementing a use case, or core services when they provide support actions such as service discovery, security, service orchestration, and Quality of Service. The approach considers the interoperability of the involved devices as one of the most important issues, and thus trust all the core services to a common Arrowhead Framework, which also provides registries containing formal descriptions of devices, systems and services. Particular support is given to the orchestration of services, to allow the creation of distributed applications that build over existing ones, for example to create demotics applications that connect to the Virtual Market of Energy that Arrowhead provides, or that interact through the publish/subscribe paradigm extended to the service-oriented world (Albano et al. 2018).

\section{Architecture for health management, prognostics and collaborative decision making}

The contemporary presence of Ethernet-based solution and distributed computational power in the industrial automation domain is pushing for the productively implementation and deployment of CPS, as confirmed by the several initiatives - such as Reference Architecture for Industry 4.0 (RAMI4.0) and Industrial Internet Consortium - Industrial Internet Reference Architecture (IIC-IIRA) - that have been created to support and enforce the usage/introduction of new internet-based technologies for improving manufacturing companies' flexibility, adaptability, efficiency and effectiveness. In particular, in the context of the MANTIS project, the RAMI4.0 and the IIC-IIRA have been analysed and studied with the objective of creating, developing a delivering reference models from the maintenance perspective i.e. for facilitating the implementation of predictive and proactive maintenance strategies. To do that, MANTIS focuses on the deep usage of IoT sensors and additional devices (Albano et al. 2018) attached to machine controllers to create an ecosystem at the shop floor level where physical assets and resources are virtualized and available as shop floor services in the cyber space. Moreover, edge and cloud computing design, architectural patterns and technical principles are used to create two level data processing architecture where: a) Low level (edge tier): where the extraction, transforming, loading and analysis functionalities are deployed near to the physical assets and resources to model and understand their behaviour while ensuring short-term decisions. b) High level (platform tier): where the extraction, transforming, loading and analysis functionalities are deployed on the cloud to model and understand the global behaviour of physical resources and assets typically using the results of the low-level data processing. The MANTIS approach is in line with the slow but inexorable transformation of the manufacturing systems towards complex environment characterized by aggregations of systems and organized according to a System of Systems (SoS) paradigm and where a large scale of collaboration take place at micro and macro level (Colombo et al. 2014)(Colombo et al. 2009) . In this context, micro level means collaboration between entities at edge level/tier on the contrary macro level collaboration relies collaboration between entities at system and/or platform level/tier. One of the tier is based on the Edge Computing paradigm, which is on the verge of rapid breakthrough. As cloud-based computing is particularly well suited for processing of large amount of information, the delay it introduces may not acceptable for applications requiring time-critical decision in the sub-milliseconds range. The main idea of Edge Computing is to process data as closely to the source as possible, i.e. locally. This processing can be as simple as filtering relevant data, but can also include virtualized services, like machine learning application and real-time visualizations. ****The advantages of edge can be distinguished in three main topics: a) Latency, if reaction speed is critical, latency is very important. An example might be the analysis on in-process sensors which need to be able to trigger a valve in sub-milliseconds. b) Security and privacy. Edge devices with respective computational power and hardware features can be used to set-up hardware encrypted data connections to the cloud to enable better protected pathways to the cloud. But it also enables to process data locally, preventing the requirement to send all data to the cloud, either solving privacy regulation or the potential risk of losing operation-critical data. c) Reliability of connection to the cloud is critical. An unreliable connection to the cloud would mean that (business critical) data processing halts or would be unreliable at best. Where to do the processing in the Edge. Make a decision about where to perform a specific processing is nothing obvious, there are several aspects to 
be taken into consideration, such as the device/product where the deployment will be performed, characteristics of the data at edge level, type of analytics to apply, the life-cycle of analytics, the functional perspective and design considerations. Edge data has specific characteristics. It is generated at the network edge and includes machine running data, environment data, and information system data. It features high throughput, fast flow movement, diversity, strong correlation, and high requirements on real-time analysis and processing. Compared with business big data scenarios such as Internet, smart analysis of edge data has the following characteristics and differences: a) Causal relationship vs. association relationship: Edge data is mainly targeted at smart assets that generally run with explicit input and output causal relationships, whereas business big data focuses on data association relationships. b) High reliability vs. low reliability: Industries such as manufacturing have high requirements on the accuracy and reliability of models. If the accuracy or reliability is low, property loss or even personal injury may occur. In contrast, business big data analysis generally has low requirements on reliability. It is required that the edge data analysis result be explainable. Therefore, blackbox deep learning is restricted in some application scenarios. The combination of the traditional mechanism models and data analysis methods is the innovation and application direction of smart analysis. c) Small data vs. big data: Assets such as machine tools and vehicles are designed and manufactured by people. Most data in their running process is predictable, and only data generated in abnormal or critical conditions is truly valuable. On the other hand, business big data analysis requires mass data. Nor should one forget that analytics has its own life cycle. Deployment of analytics typically consists of three steps: a) train a (predictive) analytics model, b) test and validate the model on previously unseen data, and c) deploy the model to make predictions on real (streaming) data. Analytic models can be deployed at the edge on heterogeneous computing elements, such as a CPU, GPU or FPGA. For example, FPGAs are ideal deployment targets for very low latency applications. From a functional point of view, the entire data analytics process consists of a series of specific modules that can, or will not, be deployed on the same node. Where to deploy data preprocessing, data analysis, data distribution and policy execution, and visualization and storage functionalities is an architecturesignificant decision to make, which is costly to revise. Hence, it demands for careful consideration of design alternatives and trade-offs.

\section{Use cases}

This section presents three of the near a dozen uses cases implemented in Mantis using the reference architecture presented in the previous section. Each example presents a summary of the objectives, the system implemented (sensors and platform), the data analysis techniques performed at platform level and the results.

5.1 Use case example Proactive Maintenance of Clutch Brake for Press Machines

Objective: This use case (Larrinaga et al. 2018) is concerned with analysing a clutch brake system and its components in press machines to detect the most important failure sources and be able to perform predictive maintenance in those press machines. The overall objective is to early detect internal wear of a clutch-brake. To do that, the static part of the clutch-brake is sensorized. The following conditions were monitored; Friction material slippage, Brake spring degradation, Friction material wear and Piston chamber air leakage. Sensors \& Platform: Several sensors have been place to measure; friction slippage (encoder sensor velocity and acceleration signals), line pressure, clutch brake input port pressure and chamber pressure (pressure sensors), friction material wear (pressure sensors) and piston chamber air leakage (air mass flowmeter). The architecture implementation agrees with the three-tier architecture presented in section 4 and in (Hegedus et al. 2018). Part of the data is collected and analysed locally (edge tier) in an industrial PLC. Information is also sent to a cloud platform where further analysis is conducted. Data Analysis Techniques: To support predictive maintenance of clutch-brake assets data analysis techniques and algorithms are implemented in the platform. These technologies are (1) Root Cause Analysis powered by Attribute Oriented Induction Clustering and (2) Remaining Useful Life powered by Time Series Forecasting. Results: At the edge tier, changes on the velocity and acceleration in the clutch-brake process have been detected when slippage situations occur. Additionally the pressure sensors and the model to represent the brake springs stiffness perform with an error less than a $5 \%$. The soft sensor is able to estimate the wear level of the friction material attached to the friction discs, establishing thresholds for different friction wear levels. The air mass flowmeter is able to quantify the air leakage that the whole system is having while the clutch brake is clutched. At platform level and by applying 
the Remaining Useful Life techniques, normal behaviour of the system has been modelled and anomalies have been detected (further research is required at this level).

\subsection{Use case example Proactive Maintenance of flue gas blowers in power plants}

Objective: This use cases addresses optimization of maintenance of components in a power plant. The main optimization maintenance goal is to minimize the total lifecycle maintenance costs and maximize the total lifecycle availability of those components. The use case in Mantis consisted of instrumenting a flue gas recirculation blower at a power plant with several different types of vibration sensors and creating a data collection and data storage system for condition base monitoring of rotating machines. Data collection from sensors is complemented with data from the plant process. All data is stored in the same data storage. Then the goal for data analysis and failure prediction study of the pilot focusses on rotating machines. Sensors \& Platform: The blower consists of three parts; the engine, bearing and impeller inside the impeller housing. Most common faults for any rotating machines are bearing failures, imbalance and misalignment. All of these causes have distinct vibration patterns that can be identified with proper vibration instrumentation. The instrumentation consisted of vibration sensors installed to the blower bearing and a tachometer for measuring the rotation frequency of the blower. The architecture of the platform agrees with the three-tier architecture presented in section 4 and in (Hegedus et al. 2018) but in this case the implementation used a Microsoft Azure based MIMOSA deployment as the information exchange platform. The data storage follows the Mimosa data structure presented in the OSA-CBM and OSA-EAI standards. Data Analysis Techniques: The success/failure of lifecycle optimization is measured by total plant availability and maintenance costs. However power plants are designed to operate for 25 - 40 years making lifecycle optimization difficult. Modern data collection, remote monitoring and analysis tools such as neural networks, statistical analytics or physical models allow cost effective implementation of more advanced maintenance methods on less critical components. Results: The bearing fault was detected about half a year before the change of the bearing based on manual expert analysis of the measured vibration signals. During the project the vibration was measured with manual method and two on-line detection systems which provided data to the cloud. For the development of the automatic prediction of the remaining useful life of the bearing a simulator that can provide vibration signals was developed i.e. due to the very limited amount of real data also simulated has been made available for the development of the prognosis tools.

\section{Conclusion}

The paper discussed how CPS can revolutionize maintenance, and presented architectures that were developed to support them, and real-life use cases where the CPS have provided dramatic improvement. The use cases show that different requirements can be implemented over the same reference architecture and that data analysis and decision making algorithms can and should be implemented both at the edge tier (for fast response) and at platform tier for combination of parameters and conditions from different sensors, components and systems. Future work will consider analysing these implemented systems of systems, to both provide quantitative measures of their benefit to business cases, and comparison between them in real life.

\section{Acknowledgement}

This work has been developed with the support of funds made available provided by the European Commission in the scope of ECSEL/H2020 MANTIS Research and Innovation Action (Project ID: 662189), by the Portuguese Fundação para a Ciência e a Tecnologia (FCT, I.P.) in the framework of project UID/EEA/00066/2013 PEST (Strategic Plan for Science and Technology) for the Centre of Technology and Systems (CTS), by the Finnish Funding Agency for Innovation Tekes, and by Ministerio de Industria, Energía y Turismo (Spain).

\section{References}

Acatech (2011) Cyber-Physical Systems - Driving force for innovations in mobility. Springer.

Albano M., Lino Ferreira F., Di Orio G., Maló P., Webers G., Jantunen E., Gabilondo I., Viguera M., Papa G., and Novak F., et al. (2018) Sensors: the Enablers for Proactive Maintenance in the Real World, 5th 
International Conference on Control, Decision and Information Technologies (CoDIT 2018). Thessalonoki, Greece. 10 to 13, April, 2018

Albano M, Lino Ferreira L, Sousa J, (2016) Extending publish/subscribe mechanisms to SOA applications, $12^{\text {th }}$ IEEE World Conference on Factory Communication Systems (WFCS 2016), May 3-6, 2016, Aveiro, Portyugal. pp. 1-4 DOI: 10.1109/WFCS.2016.7496528

Baheti R, Gill H, (2011) Cyber-physical systems, The Impact Control Technology, T. Samad and A.M. Annaswamy (eds.), pp. 161-166

Bytschkow D, Campetelli A, Cengarle MV, Irlbeck M, Schorp K (2014) Reference Framework for the Engineering of Cyber-Physical Systems: A First Approach

Cândido GM (2013) Service-oriented architecture for device lifecycle support in industrial automation

Candido G, Jammes F, de Oliveira JB, Colombo AW (2010) SOA at device level in the industrial domain: Assessment of OPC UA and DPWS specifications, 8th IEEE International Conference on Industrial Informatics (INDIN), pp. 598-603.

Cavalieri S, Pezzotta G (2012) Product-Service Systems Engineering: State of the art and research challenges, Computers in Industry vol. 63, no. 4, pp. 278-288

Cengarle M, Bensalem S, McDermid J, Passerone R, Sangiovanni-Vincetelli A, Torngren M, (2013) Characteristics, capabilities, potential applications of Cyber-Physical Systems: a preliminary analysis, D2.1

Colombo, A., Bangemann, T., Karnouskos, S., Delsing, J., Stluka, P., Harrison, R., Jammes, F., Lastra, J.L. (Eds.), 2014. Industrial Cloud-Based Cyber-Physical Systems: The IMC-AESOP Approach, 2014 edition. ed. Springer, New York.

Colombo, A.W., Karnouskos, S., 2009. Towards the factory of the future: A service-oriented cross-layer infrastructure, ICT Shaping the World: A Scientific View, European Telecommunications Standards Institute (ETSI) John Wiley Sons 65, 81.

Delsing, J (2017) The Arrowhead Framework architecture, IoT Automation: Arrowhead Framework, CRC Press, pp 45-91.

Di Orio G, Cândido G, Barata J (2015) The Adapter module: A building block for Self-Learning Production Systems, Robotics and Computer-Integrated Manufacturing, vol. 36, pp. 25-35

Hadlich T (2006) Providing device integration with OPC UA, in Industrial Informatics, IEEE International Conference on Industrila Informatics, pp. 263-268.

Hegedus C., Varga P. and Moldovan I. «The MANTIS architecture for proactive maintenance.» 5th International Conference on Control, Decision and Information Technologies CODIT 2018 Thessaloniki (Greece), 2018.

Larrinaga F., Fernandez-Anakabe J., Zugasti E., Garitano I., Zurutuza U., Anasagasti M., Mondragon M. «Implementation of a Reference Architecture for Cyber Physical Systems to support Condition Based Maintenance.» 5th International Conference on Control, Decision and Information Technologies CODIT Thessaloniki (Greece), 2018.

Leitão P, Colombo AW, Karnouskos S (2016) Industrial automation based on cyber-physical systems technologies: Prototype implementations and challenges, Computers in Industry, vol. 81, pp. 11-25

OASIS (2009) OASIS Web Services Dynamic Discovery (WS-Discovery) Version 1.1 [Online], Available: http://docs.oasis-open.org/ws-dd/ns/discovery/2009/01.

Rajaraajeswari S, Selvarani R, Raj P (2016) Integration Approaches for the Internet of Things (IoT) Era in Connectivity Frameworks for Smart Devices, Z. Mahmood, Ed. Springer International Publishing, pp. 117146.

Sanislav T, Miclea L (2012) Cyber-Physical Systems - Concept, Challenges and Research Areas, Journal of Control Engineering and Applied Informatics, vol. 14, no. 2, pp. 28-33

Steering Committee (2013) Strategic R\&D Opportunities for 21st Century Cyber-Physical Systems, Connecting computer and Information systems with physical world. 